patients had hypovitaminosis D. In addition, an associated iron deficiency anemia was detected in $50 \%$ of the cases, biological stigma of digestive malabsorption in $40 \%$ of the cases and renal insufficiency in $15 \%$ of the cases. Standard radiographs showed diffuse demineralization in $90 \%$ of cases, Looser-Milkman streaks in $40 \%$ of cases and fractures in $30 \%$ of cases. Bone scintigraphy among 8 patients revealed diffuse hyperfixation in 4 cases $(50 \%)$, localized hyperfixation in 3 cases $(37 \%)$ and non conclusive images in only one case $(12.5 \%)$. Bone densitometry performed in 5 patients showed secondary osteoporosis in $80 \%$ of cases. The diagnosis of osteomalacia was retained in 2 cases, celiac disease in 5 cases, renal insufficiency in 1 case, phosphate diabetes in 3 cases including Fanconi syndrome, hypophosphatasia in one case and tubular involvement as part of ankylosing spondylitis In one case. Etiology was a neoplastic cause in 4 cases including multiple myeloma. All the patients had a vitamin-calcium treatment (calcium intake between $500 \mathrm{mg}$ and $2 \mathrm{~g}$ per day with an average of $2.5 \mathrm{cp} / \mathrm{d}$, phosphorus in 3 cases and an etiological treatment in 3 cases. The resection of the tumor was the reason behind a clinical-biological improvement in the case of thyroid tumors. The outcome was favorable in the short term for all cases.

Conclusions: Osteomalacia is a generalized benign osteopathy, essentially linked to a deficiency in vitamin $D$ often unrecognized. The diagnosis is simple and the treatment is easy, but the ignorance of the etiological forms can be responsible for an unfavorable evolution.

Disclosure of Interest: None declared

DOI: 10.1136/annrheumdis-2017-eular.6986

\section{AB0877 APPLICATION OF GOUT DIARY IN HEALTH EDUCATION OF GOUT PATIENTS}

H. Miao, L. Zhang, G. Zhang, K. Xu. Rheumatology, Shanxi dayi hospital, Taiyuan, China

Background: Gout is a heterogeneous disease characterized by increased levels of blood uric acid. It is difficult to achieve a comprehensive and effective control of gout only by drugs, drug therapy must be combined with health education, diet and lifestyle changes. However, most of the gout patients had wrong thinking about their disease, diet and drug therapy. The status of China's health education in gout patients:the research teams were dispersed and unstable, the content of health education needed to be further refined, the models of health education were very simple.Based on the traditional models of health education,the purpose of this study is to explore the effect of gout diary in gout patients, by recording gout diary in order to improve their daily life behaviors and related laboratory indexes, aim to provide a new method for health education of gout patients.

Objectives: In order to explore the effect of gout diary,we applied the gout diary in gout patients.

Methods: From January 2015 to December 2015,110 cases of gout hospitalized patients were randomly divided into study group and control group $(n=55)$. The control group used traditional education models:patient education forum,gout handbook,patient group discussion,etc.Based on the control group,the research group used not only the traditional education models, but also added gout diary, the patient can record the diary by themselves. In order to evaluate the effect of gout diary,we used questionnaires and related laboratory indexes.

Results: The research group had significant improvement in laboratory indexes, healthy behaviors and life style $(P<0.05)$,such as quit smoking, quit drinking, according to the doctors' suggestions, pay more attention to their blood uric acid and other laboratory indexes, see the doctor regularly, maintain ideal body weight,exercise regularly,working and resting regularly,keeping happy mood. Conclusions: Apply the gout diary in gout patients, we can achieve a perfect effect.

References:

[1] Li Yun Zhang, MD, H. Ralph Schumacher, Hou Heng Su, et al.Development and Evaluation of a Survey of Gout Patients Concerning Their Knowledge About Gout. Journal of Clinical Rheumatology[J]. 2011, 8,17(5):1-7.

[2] Glazebrook KN, Guimaraes LS, Murthy NS, et al. Identification of Intraarticular and Periarticular Uric Acid Crystals with Dual-Energy CT[J]. Initial Evaluation Radiology. 2011, 261(2):516-524.

[3] Roddy E, Doherty M. Epidemiology of gout[J]. Arthritis Res Ther, 2010,12(6):223.

[4] Zhu Y, Pandya BJ, Choi HK. Comorbidities of gout and hyperuricemia in the US general population: NHANES 2007-2008[J]. The American Journal of Medicine, 125(7), 2012:679-687.

[5] Choi HK, Curhan G. Independent impact of gout on mortality and risk for coronary heart disease. Circulation, 2007,116(8):894-900.

[6] Rees F, Jenkins W, Doherty M. Patients with gout adhere to curative treatment if informed appropriately:proof-of-concept observational study[J]. Ann Rheum Dis, 2013,72(6):826-830.

[7] Pullen C, Walker SN, Fiandt K. Determinants of health-promoting lifestyle behaviors in rural older women[J]. Fam Community Health, 2001,24(2):49-72.

Acknowledgements:

Disclosure of Interest: None declared

DOI: 10.1136/annrheumdis-2017-eular.3370

\section{AB0878 EFFECT OF DIALYSIS AND LOW DOSE URATE-LOWERING ON SERUM URATE LEVELS IN PATIENTS WITH GOUT}

I. Urionagüena ${ }^{1,2}$, S.P. Chinchilla ${ }^{2,3}$, G. Garcia Erauzkin ${ }^{4}$, M.L. Muñiz-Gomez ${ }^{4}$, F. Pérez-Ruiz ${ }^{1,2,3}$. ${ }^{1}$ Rheumatology Division, Cruces University Hospital; ${ }^{2}$ BioCruces Health Research Institute, Barakaldo, Biscay; ${ }^{3}$ University of the Basque Country, Basque Country; ${ }^{4}$ Nephrology Division, Cruces University Hospital, Barakaldo, Biscay, Spain

Background: The effect of dialysis on urate levels (sUA) in patients with gout has not been well characterized. No prospective series are available from the literature, and no recommendation has yet been made by either EULAR or ACR on the topic.

Methods: Exploratory, observational, prospective study. Patients with crystalproven gout and at least one flare the previous year to entrance into dialysis program, either hemodialysis (HD) or home peritoneal dialysis (PD), were followed-up. No urate-lowering drug was prescribed for the first 3-month period, but low-dose allopurinol ( $50 \mathrm{mg} /$ day) was to be prescribed from 6-month visit onwards if tophi (any subcutaneous or ultrasonographic $>10 \mathrm{~mm}$ longest diameter) were present or febuxostat in case allopurinol was not clinically suitable. sUA levels previous to the first dialysis procedure were recorded, along with

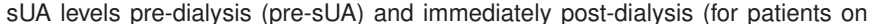
hemodialysis) sUA (post-sUA) were obtained during follow-up at 3,6 , and 12 months of follow-up. No prophylaxis was prescribed, and only adrenocorticotropic hormone $(\mathrm{ACTH})$ analogue or corticosteroids were to be used for the treatment of flares.

Results: 17 patients studied to date: 12 men, mean age 69 (IQ range 62-78). Time from onset of gout was 7years (IQR 1-10) pre-dialysis, and 2 patients entered into dialysis due to prolonged use of NSAIDs because recurrent, longstanding gout over 20 years. Allopurinol $(n=6)$ and febuxostat $(n=2)$ had been prescribed previously to dialysis procedures. ULT was prescribed to 10 patients: 4/4 patients on PD and 6/13 on HD as per in methods; patients treated were prescribed allopurinol $50 \mathrm{mg} /$ day but one who showed HLA-B58 antigen and was treated with febuxostat $80 \mathrm{mg}$ every 3 days. After 12 months 5 patients were lost for follow-up: 2 underwent a renal transplant, 3 were dialyzed at private centers. Follow-up ranged from 12 to 30 months.

There was a significant reduction in sUA at 3-month $(\Delta-2.6 \mathrm{mg} / \mathrm{dl})$, compared to baseline sUA. Reduction of sUA from 3-6-month and 3-12 month was greater in patients treated with ULT (Table).

The rate of flares at 3-month was similar in patients on HD (5/13) than in patients on PD (2/4), only one flare was reported at 6 month follow-up visits, none afterwards. No serious side effect was observed.

Table 1. Change in pre-sUA and post-sUA (mg/dl) globally and with interventions

\begin{tabular}{lccccccc}
\hline Patients/sUA & $\begin{array}{c}0 \\
\text { month }\end{array}$ & $\begin{array}{c}\text { 3rd } \\
\text { month }\end{array}$ & $\begin{array}{c}\text { PostHD- } \\
\text { 3rd month }\end{array}$ & $\begin{array}{c}\text { 6th } \\
\text { month }\end{array}$ & $\begin{array}{c}\text { PostHD- } \\
\text { 6th month }\end{array}$ & $\begin{array}{c}\text { 12th } \\
\text { 12th month }\end{array}$ \\
\hline All $(\mathrm{N}=17)$ & 9.8 & 7.2 & 2.8 & 5.8 & 3.1 & 5.5 & 1.2 \\
Not treated $(\mathrm{N}=10)$ & 9.4 & 6.2 & 3.1 & 5.7 & 3.2 & 5.8 & 1.2 \\
Treated from 6-month $(\mathrm{N}=7)$ & 10.0 & 7.7 & 2.7 & 5.8 & 3.0 & 5.3 & 1.2 \\
PD & 10.2 & 8.0 & & 6.0 & & 5.0 & \\
HD & 9.7 & 6.9 & 2.8 & 5.7 & 3.0 & 5.7 & 1.2 \\
\hline
\end{tabular}

Conclusions: In this exploratory study, dialysis is effective in reducing sUA levels. In hemodialysis, both sUa and postHD-sUA may be needed to better estimate the effect of interventions on SUA. Low dose ULT seems to be effective and may be considered to properly control sUA, but only to be considered in patients with severe (tophaceous) gout.

Disclosure of Interest: I. Urionagüena: None declared, S. P. Chinchilla: None declared, G. Garcia Erauzkin: None declared, M. L. Muñiz-Gomez: None declared, F. Pérez-Ruiz Consultant for: Amgen, Ardea, AstraZeneca, Gruenenthal, Menarini, Speakers bureau: AstraZeneca, Gruenenthal, Menarini

DOI: 10.1136/annrheumdis-2017-eular.2980

\section{AB0879 AN INTERNET TRIAL OF CHERRY EXTRACT VS. DIET MODIFICATION: PATIENT CHARACTERISTICS, GOUT SEVERITY AND DIET CHARACTERISTICS}

J. Singh ${ }^{1}$, G. Mcgwin ${ }^{2}$. ${ }^{1}$ Rheumatology; ${ }^{2}$ Biostatistics, University of Alabama at Birmingham, Birimingham, United States

Background: Gout is often treated with life-long pharmacological therapies. Patients are interested in non-pharmacological interventions as adjunct to these therapies. To our knowledge, non-pharmacological have not been studied in detail.

Objectives: To describe the baseline characteristics of patients with gout enrolled in an Internet pilot study comparing the benefits and harms of diet modification to cherry extract intake.

Methods: Patients were enrolled over an 8-month period using the Internet. Diagnosis of gout was confirmed by contacting their health care provider. We describe the baseline characteristics of patients with physician-confirmed gout, who were randomized in the Internet gout study.

Results: We randomized 83 participants in an Internet gout pilot study, randomized to diet modification $(n=43)$ or cherry extract $(n=40) ; 3$ patients withdrew before receiving intervention. The mean age of study participants was 56 years, (SD, 